\title{
DisTRIBUTED COVERAGE AND CONNECTIVITY Preserving AlgorithM With SuPPORT OF DifFerent SENSINg Coverage DegreEs IN3D MOBILE SENSOR ACTOR NETWORKS
}

\author{
Mohammad Javad Heydari ${ }^{1}$ and Saeid Pashazadeh ${ }^{2}$ \\ ${ }^{1}$ Faculty of Electrical and Computer Engineering, University of Tabriz, Tabriz, Iran \\ Mj. Heydari89 ems.tabrizu.ac. ir \\ ${ }^{2}$ Faculty of Electrical and Computer Engineering, University of Tabriz, Tabriz, Iran \\ Pashazadeh@tabrizu.ac.ir
}

\begin{abstract}
Given a 3D space where should be supervised and a group of mobile sensor actor nodes with limited sensing and communicating capabilities, this paper aims at proposing a distributed self-deployment algorithm for agents to cover the space as much as possible by considering non-uniform sensing coverage degree constraint of environment while preserving connectivity. The problem is formulated as coverage maximization subject to connectivity and sensing coverage degree constraint. Considering a desired distance between neighbouring nodes, an error function which depends on pairwise distance between nodes is described. The maximization is encoded to an error minimization problem that is solved using gradient descent algorithm and will yield in moving sensors into appropriate positions. Simulation results are presented in two different conditions that importance of sensing coverage degree support of environment is very high and is low.
\end{abstract}

\section{KEYWORDS}

Mobile Sensor Networks, Coverage, Connectivity, Quality of Services, Sensing Coverage Degree

\section{INTRODUCTION}

Wireless sensor actor networks (WSANs), can be deployed in three dimensional (3D)spacein many applications such as aerial defence systems [1], underwater WSNs for Ocean sampling and tsunami warnings [2-6], intrusion detection systems, aerosphere pollution monitoring [7], etc. Maximal Coverage of the target volume and having a connected network are important criteria in terms of the Quality of Service (QOS) required for WSNs. In this paper, considering importance of coverage and connectivity in $3 \mathrm{D}$ wireless sensor networks a distributed maximizing coverage algorithm is proposed.

The network consists of mobile agents deployed to perform collaborative monitoring tasks over a given volume.. The problem is modelled as maximization which is solved completely distributed. In fact each agent tries to maximize its sensing volume while preserving connectivity and having high quality communication links. First of all, Knowing that distance of two communicating nodes have great impact on energy consumed for transmission, quality of their connection and 
total coverage of network and regarding the remaining energy, required quality of coverage and connectivity due to application and required link strength depend on environment situation, an optimal distance for two neighbouring nodes was assumed. Then each agent knowing distance of its neighbours defines an error function and tries to decrease the error by changing its positions through gradient descent algorithm. In other words agents try to set their distance to their neighbours something near desired value by changing their positions. After obtaining optimum positions, all agents will go to optimal points which yields lower using of energy in deployment and moving.

It is well-known that centralized coordination algorithms have potential to have bottleneck and suffer from computational complexity and lack of adaptation, thus distributed algorithms are preferred. In this type of solutions although there is no centralized coordinator in the network, covered area by the network is increasing from one step to another.

Our contributions in this paper are as follows: 1) Proposedalgorithm is completely distributed and there is no need to having extra information. 2) As computation consumed much lower energy in comparison with moving, the approach is using lower energy and thus the lifetime of network would increase. 3) The parameter of network is adjustable and can vary depending on application. In fact, coverage can be increased by increasing the desired distance of two nodes and by decreasing it the quality of connections and usually number of links will be increased which means a better connectivity and lower power consumption. The reminder of this paper is organized as follows: The next section is covering related work. The considered system model and the problemare described in section 3. Section 4 and 5 discuss and analyse the proposed approach. Simulation results and performance evaluation are discussed in section 6.section 7 concludes the paper.

\section{RELATED WORK}

Deferent approaches for providing maximum coverage are proposed in manyliteratures in context of multi-robot networks, mobile sensor networks and wireless sensor and actor networks. In most of papers, the network is considered in 2D space where mobile node deployed randomly and can move around to adjust their positions after initial deployment in order to reduce their coverage overlaps and maximize area coverage while preserving connectivity.

Many presented approaches reducing the 3D geometry to the 2D [8-12]. Different modelling and strategies such as virtual forces, optimization and predefined pattern was proposed. In [13] Megerian and Potkonjakmodelled the coverage problem in wireless sensor networks as Integer Linear Programming (ILP) and solved the problem using a greedy algorithm. Similarly, Nakamura et al [14] used ILP modelling and solve it with the commercial optimization package CPLEX [15]. Yiannis and Tzes in [16] and[17] modelled the coverage problem as a constrained maximization. Our proposed algorithm in [18] is mainly inspired from the approaches used to model the problem in [16] and [17] and current paper develops the approach that is used in [18].In previous paper, we assumed equal importance for deferent points in the environment while this paper uses a non-uniform density function. This means that points of 3D space have different importance level and some points need higher sensing coverage degree in comparison with other areas.

In one of the other group of coverage improvement algorithms such as [19], pattern-based strategies are presented in 2D and 3D space. In such algorithms target locations that can provide both coverage and network connectivity requirements are computed based on a predefined pattern and mobile nodes will deploy in the target positions. Alam and Haas [20] proposed a deployment 
pattern that generates the Voronoi tessellation of truncated octahedron in 3D space. Achieving full-coverage and k-connectivity in three-dimensional space, some lattice was proposed in [21] and[22].Wu et al [23] used Delaunay Triangulation to solve the coverage problem and finding the position of sensors in the environment. Vieira et al [24] use computational geometry and graph theory to find a solution. In virtual force-based node movement strategies [25-27], mobile nodes affect each-others. In detailed form, two mobile nodes expel each other if their distance is close than a threshold or attract each other if their distance is too far away. Other types of forces such as attractive force of boundaries and the repulsive forces of obstacles also can be considered. The total force is vector addition of all forces which specifies the direction and distance of mobile node movement. In [28] and[29], the coverage problem is modelled as an optimization problem and is solved through evolutionary algorithms PSO and ant colony optimization respectively.

\section{Problem Formulation}

As mentioned in advanced, in this paper previous proposed approach in [18] is developed. So similar to [18], let assume that network includes set of mobile homogenous agents denotedbyA $=$ $\left\{\mathrm{a}_{1}, \mathrm{a}_{2}, \ldots, a_{n}\right\}$. Nodes are distributed with uniform distributionin 3D space called volume of interest that we represent by $v \subset \mathrm{R}^{3}$. Let assume that all nodes are location aware and each node knows its position $\mathrm{p}_{\mathrm{i}} \mathrm{using}$ a GPS or other node localization approaches and is aware ofpositions of its one-hop neighbours. All agents equipped with wireless transceiver and the transmission range of all agents is equal and is denoted by $R_{c}$ and is much less than dimension of volume of interest. The communication range of a node is defined as maximum Euclidean distance that its radio signals effectively can reach to other agents. Considering communication range of each agent, communication volume of agent $i$ is denoted by $c_{i}$ and is a sphere centred in agent position i.e.

$$
c_{i}=\left\{p \in R^{3} \mid\left\|p-p_{i}\right\| \leq R_{c}\right\}, i \in I_{n}
$$

where $\|$.$\| stands for standard Euclidean norm and \mathrm{I}_{\mathrm{n}}$ is defined according to following equation:

$$
I_{a}=\{i \in \mathbb{N} \mid i \leq a\}, a \in \mathbb{N}
$$

Similarly, the sensing volume of agent $i$ is denotedby $s_{i}$ is a sphere of radius $R_{S}$, having the sensor node at its centre i.e.

$$
s_{i}=\left\{p \in R^{3} \mid\left\|p-p_{i}\right\| \leq R_{s}\right\}, i \in I_{n}
$$

Also it is assumed that sensing and transmission ranges of all agents are equal,but transmission range is greater than sensing radius.Let the density function $\phi: v \subset R^{3} \rightarrow \mathbb{R}^{+}$demonstrate probability of occurring an event in an arbitrary pointp $\in v$. This function explains importance of each point in 3D space of volume of interest. Some points require more sensing coverage degree in comparison to other points. The assumed density function in this paper is 3D Gaussian function which is defined by Equation (4). As it can be seen in Figure 1, the density function peaks at its centre and the importance of points decrease as they get farther. The centre location and other parameters determine the shape of function and peace of decrease in deferent dimensions. Assuming an arbitrary point, $p(x, y, z) \in R^{3}$ in the space, and $p_{c}\left(x_{c}, y_{c}, z_{c}\right) \in R^{3}$ as centre of Gaussian function, the following equationrepresents our assumed density function:

$$
\phi(p)=e^{\left(-\frac{\left(x-x_{c}\right)^{2}}{\sigma_{x}^{2}}-\frac{\left(y-y_{c}\right)^{2}}{\sigma_{y}^{2}}-\frac{\left(z-z_{c}\right)^{2}}{\sigma_{z}^{2}}\right)}
$$

Let Assume that the volume of interest is much greater than sensing volume of an individual agent and variation of density function occurs smoothly. So,each sensing volume can be 
estimated by its center point. In fact importance of agent location is considered as mean of covered volume of all points of its sensing sphere.Another important function is $f_{i}: v \subset R^{3} \rightarrow$ $\mathbb{R}^{+}$which is determined by following equation:

$$
f_{i}(p)= \begin{cases}1 & \text { if } p \in s_{i} \\ 0 & \text { if } p \notin s_{i}\end{cases}
$$

This function determines if agent $i$ covers the point $p$ or not.
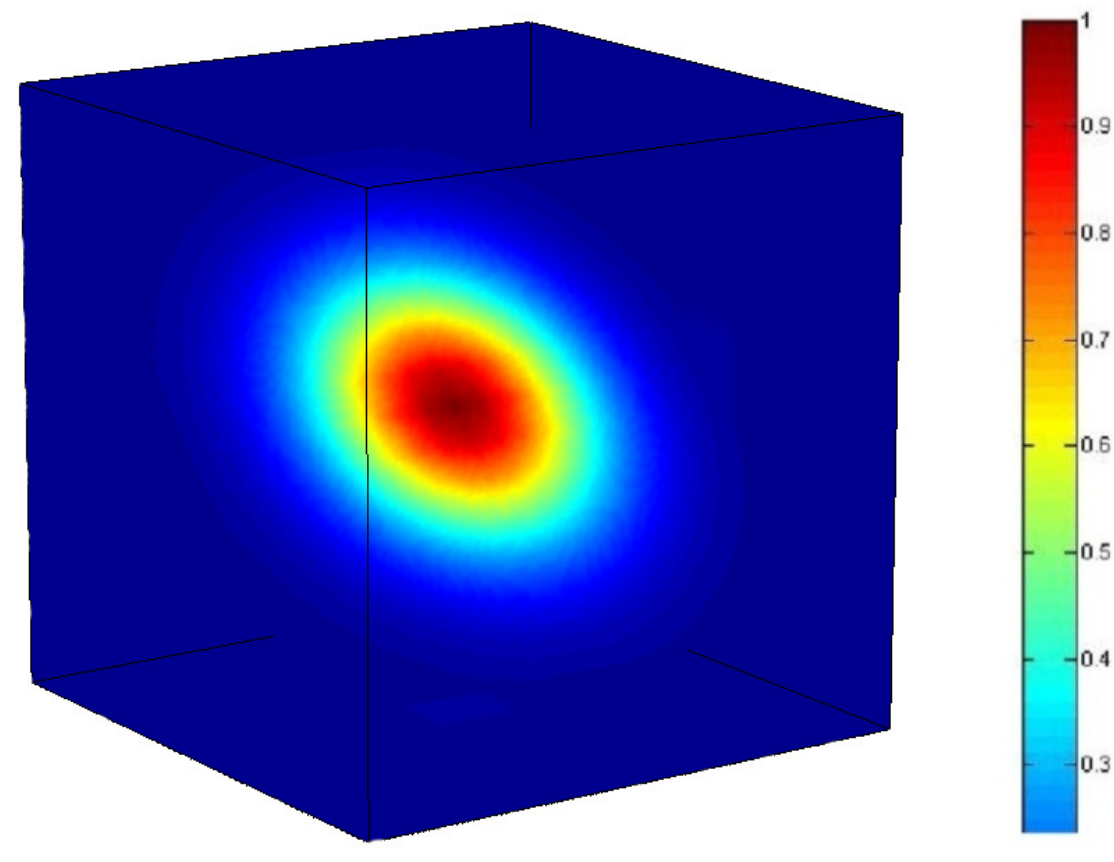

(a)
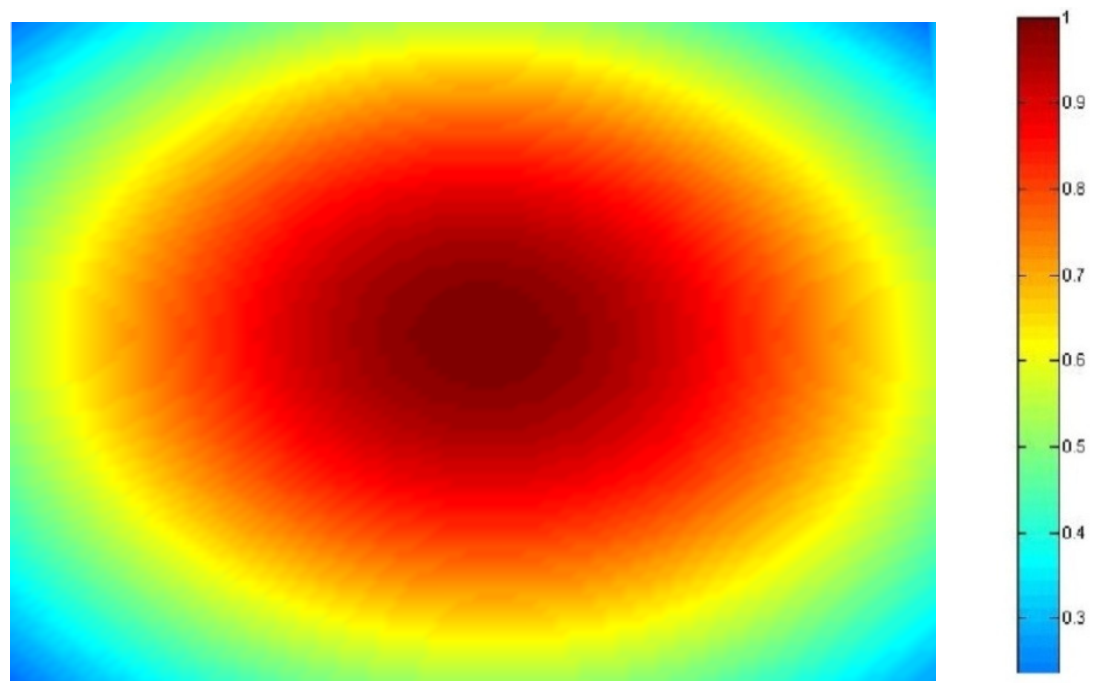

(b)

Figure 1. (a) Cross section of density function in 3D view (b) Lateral view of cross section of density function. 
Considering aforementioned functions and notations the main objective of this paper can be expressed as proposing a distributed algorithm to maximize following function $\mathcal{C}$ :

$$
\mathcal{C}=\bigcup_{i=1}^{n} \int_{v} f_{i}(p) \phi(p) d p, i \in I_{n}
$$

If the position metric considered as $P=\left(p_{1}, p_{2}, \ldots, p_{n}\right)$ the maximization can be defined as following constrained optimization:

$$
\begin{gathered}
\text { find } P: \\
\text { maximize } \mathcal{C} \\
\text { subject to: not losing connectivity }
\end{gathered}
$$

The Voronoi diagram for a set of points in a given space $R^{d}$ is the partitioning of that space into regions such that all locations within any one region are closer to the generating point than to any other. In three dimensions, a Voronoi cell is a convex polyhedron formed by convex faces, as shown in the Figure 2.The $i$ th node partition or $i$ th node Voronoi cell is defined as follows:

$$
v_{i}=\left\{p \in v,\left\|p-p_{i}\right\| \leq\left\|p-p_{j}\right\|, \forall j \in I_{N}, j \neq i\right\}
$$

Limited Voronoi cell of $i$ th node is defined usingEquation (8) and is depicted in Figure 2 as follows:

$$
v_{i}^{l}=v_{i} \cap s_{i}, i \in I_{n}
$$

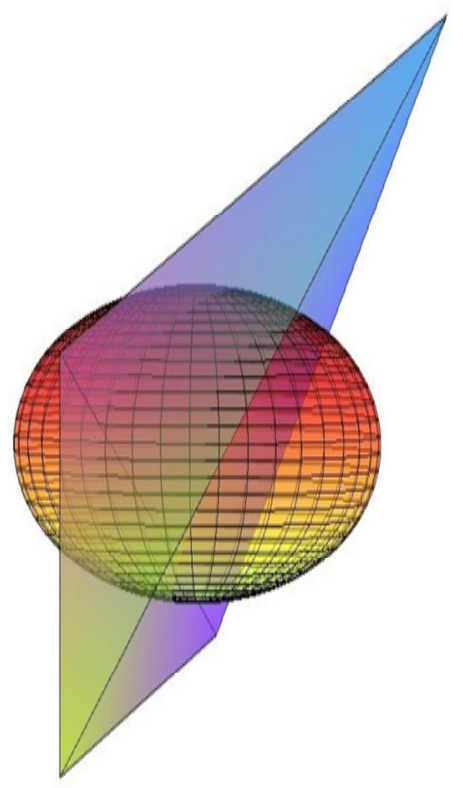

Voronoi Cell

Limited Voronoi Cell

Sensing region

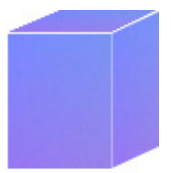

Figure 2. Demonstration of a Voronoi cell [18]. 
Denoting $N_{i}$ asone-hop neighbour of $i$ th agent, the set of communication linksof $i$ th node is defined as:

$$
L_{i}=\left\{(i, j) \mid j \epsilon N_{i}\right\}, \quad i, j \in I_{n}
$$

Using similar approach to [18] the Equation 7 could be expressed as:

$$
\begin{gathered}
\text { find } p_{i}: \\
\text { maximize } v_{i}^{l} \\
\text { subject to: } L_{i} \neq \varnothing
\end{gathered}
$$

In fact the maximization problem defined by Equation (14) can be divided into sub-problems which are solvable through local information and in a distributed manner.

\section{PROPOSED APPROACH}

Considering Equation (11), each agent tries to increase volume of its Voronoi cell without losing connectivity with the others while trying to keep itself closer to areas that requires more sensing coverage degree. In this section a parallel constrained maximizing algorithm running in all agents is proposed.

To perform the algorithm, first desired distance between two neighbours $(\alpha)$ is specified. The value of $\alpha$ would determine importance of coverage and connectivity, such that by increasing or decreasing $\alpha$ coverage or connectivity is made more important in other word having a greater $\alpha$ means increasing coverage of each node and thus increasing total coverage of network and decreasing $\alpha$ means having lower distance between nodes, so having a more compact and connected network. As transmission is one of energy-consuming act of network, it should also be noted that increasing or decreasing $\alpha$ will affect the energy consumption of network since decreasing distance of two neighbour nodes means increasing signal power and decreasing node distance means decreasing power used for transmission [18].

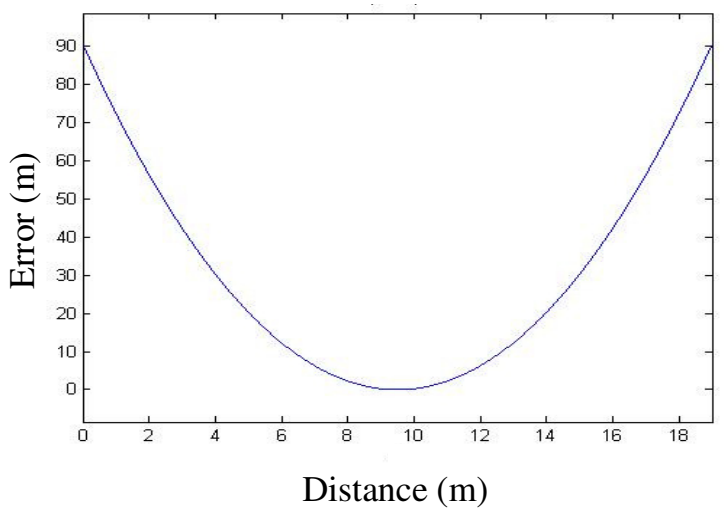

Figure 3. Shape of error function

By determining $\alpha$, a neighbourhooderror function easily could be obtained. The error functions assign an error to each distance. In fact knowing the distance between nodes $i$ and $j$ represented by $d(i, j)$, the function assigns an error to $d(i, j)$. The amount of error is zero when $d(i, j)$ equals $\alpha$ and will increase as the distance become more or less. The error function is plotted in Figure 3 $(\alpha=9.5 \mathrm{~m})$. 
Denoting position of $i$ th node or $i$ th column of $P$ as $p_{i}$, obtaining the following equations seems straightforward.

$$
p_{i}\left(x_{i}, y_{i}, z_{i}\right) \in R^{3}
$$

Let assume

$$
\Delta p_{i j}=p_{i}-p_{j}
$$

And

$$
d_{i j}=\sqrt{\Delta p_{i j}{ }^{T} \Delta p_{i j}}
$$

The error between nodes $i$ and $j$ is difference of their distance from predetermined threshold $\alpha$.

$$
e_{i j}=d_{i j}-\alpha
$$

So the neighbourhood error of $i$ th node is as follows:

$$
E_{N_{i}}=\frac{1}{\left|N_{i}\right|} \sum_{j \in N_{i}} e_{i j}^{2}
$$

And the positionalerror of $i$ th node is as follows:

$$
E_{p_{i}}=1-e^{\frac{-d_{c}^{2}}{\sigma^{2}}}
$$

Where $d_{c}$ represents the distance from centre of Gaussian. So the total error of $i$ th node is:

$$
E_{i}=A E_{N_{i}}+B E_{p_{i}}
$$

We call parameter $A$ as spreading coefficient. It represents the importance of maximizing coverage while preserving connectivity.Parameter $B$ represents the importance of supporting required sensing coverage degree and we call it density coefficient. In other words, this parameter represents tendency of agents to get closer to areas that requires more sensing coverage degree. By appropriate adjustment of these parameters, non-uniform sensing coverage degree on the environment could be supported. In asymptotic case, if the parameter $B$ tends to zero and parameter $A$ set to one, agents have no tendency to get closer to points that requires more sensing degree and only spread in the environment such that supports sensing degree one for all points of the environment. We name this as spreading case in future. 
If the parameter $B$ set to one and parameter $A$ set to zero the agents act like consensus state. In this conditions, Agents tends to focus in areas that requires more sensing coverage degrees. We name this case as aggregating case in remaining of the paper.

Position of $i$ th node is changed based onfollowing equation:

$$
p_{i}(t+1)=p_{i}(t)-\eta \frac{\partial E_{i}}{\partial p_{i}}
$$

To getting derivate from $E_{N_{i}}$ we have:

$$
\begin{gathered}
\frac{\partial E_{N_{i}}}{\partial p_{i}}=\frac{1}{\left|N_{i}\right|} \sum_{j \in N_{i}} 2 e_{i j} \frac{\partial e_{i j}}{\partial p_{i}} \\
\frac{\partial E_{N_{i}}}{\partial p_{i}}=\frac{1}{\left|N_{i}\right|} \sum_{j \in N_{i}} 2 e_{i j} \frac{\partial e_{i j}}{\partial d_{i j}} \frac{\partial d_{i j}}{\partial p_{i}} \\
\frac{\partial E_{N_{i}}}{\partial p_{i}}=\frac{1}{\left|N_{i}\right|} \sum_{j \in N_{i}} 2 e_{i j} \frac{\partial e_{i j}}{\partial d_{i j}} \frac{\partial d_{i j}}{\partial \Delta p_{i j}} \frac{\partial \Delta p_{i j}}{\partial p_{i}} \\
\frac{\partial d_{i j}}{\partial \Delta p_{i j}}=1 \\
\frac{\partial d_{i j}}{\partial \Delta p_{i j}}=\frac{\Delta p_{i j}}{d_{i j}} \\
\frac{\partial \Delta p_{i j}}{\partial p_{i}}=1
\end{gathered}
$$

Using the above equations we get:

$$
\frac{\partial E_{N_{i}}}{\partial p_{i}}=\frac{1}{\left|N_{i}\right|} \sum_{j \in N_{i}} 2 e_{i j} \frac{\Delta p_{i j}}{d_{i j}}
$$

To getting derivate from $E_{p_{i}}$ we have:

$$
\frac{\partial E_{p_{i}}}{\partial p_{i}}=\frac{2 d_{c}}{\sigma^{2}} e^{\frac{-d_{c}^{2}}{\sigma^{2}}}
$$

By substituting Equation (26) and(27) inEquation (19), the following equation is obtained:

$$
p_{i(t+1)}=p_{i(t)}-2 \eta\left(\frac{A}{\left|N_{i}\right|} \sum_{j \in N_{i}} e_{i j} \frac{\Delta p_{i j}}{d_{i j}}+B \frac{d_{c}}{\sigma^{2}} e^{\frac{-d_{c}^{2}}{\sigma^{2}}}\right)
$$

Agents by using Equation (28) can change their positions in each time step such that $\mathcal{C}$ is a nondecreasing function of time. The modification will halt when:

$$
p_{i(t)}-p_{i(t+1)}<\epsilon
$$




\section{Algorithm ANALYSIS}

In this section the algorithm is investigated. The algorithm will be verified in maximizing coverage especially around the centre and not losing connectivity. If the minimum distance between two neighbours is set to $\alpha$, the volume of limited Voronoi cell of $i$ th node can be estimated by a sphere with radius equal to $\alpha / 2$ :

$$
v_{i}^{l} \geq \frac{1}{6} \pi \alpha^{3}
$$

Considering Equations (30) and (6) the following equations will tie $\alpha$ in total coverage of network.

$$
\begin{gathered}
\mathcal{C} \geq \bigcup_{i=1}^{n} \frac{1}{6} \pi \alpha^{3} \\
\mathcal{C} \geq \frac{n}{6} \pi \alpha^{3}
\end{gathered}
$$

So by increasing $\alpha$, total coverage of network will be increased and vice versa.Number of agent in a determined volume is called density of agent which is defined by following equation:

$$
\lambda=\frac{1}{\frac{1}{6} \pi \alpha^{3}}
$$

So by decreasing $\alpha$, the agents density around the centre will be increased and vice versa. Due to using Exponential model for communication links decreases the distance between two nodes results better quality of connections or higher connectivity. The exponential model is plotted in Figure4for parameter $\sigma^{2}=0.5$. The vertical column represent remaining portion of signal power versus distance from the node.

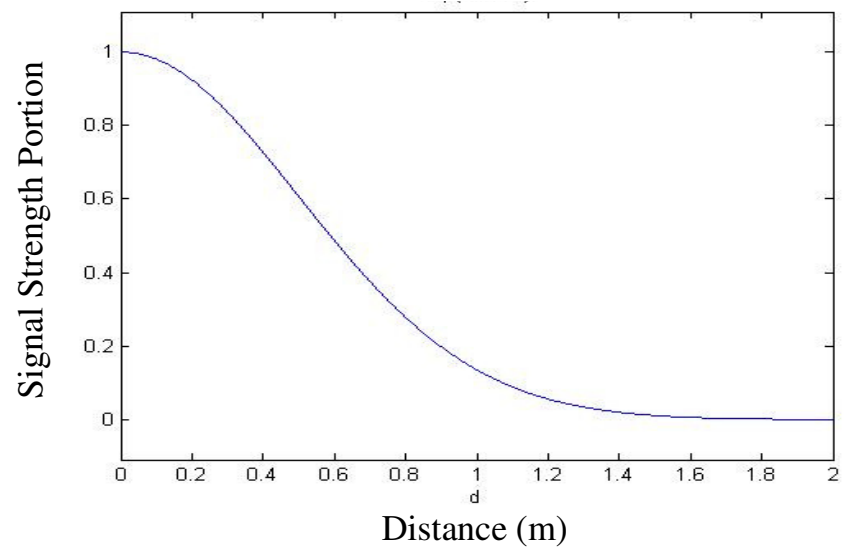

Figure 4. Exponential model of connection

It is so clear that adjusting $\alpha$ lower than communication range prevent links from failing.By considering the convexity of error functions and lack of any local minima, the gradient descent algorithm will guarantee to find a position in which global minimum error occurred. We can conclude that by setting $\alpha$ lower than $R_{C}$ the network will stay connected and by considering $\alpha$ near sensing radius the sensing volumes are maximized and by decreasing $\alpha$ the density of nodes are increase around the centre. So in determining above-mentioned parameters should be considered. 


\section{Simulation RESUltS}

Let assume a networkwhich includes 10agents deployed randomly with uniform distribution on the space $v \subset \mathrm{R}^{3}$. During simulation, the covered volume is increasing and agents get near to centre. Two series of simulations is done as follows: considering a network with 1)high positional error coefficient and 2) with low positional error coefficient. The simulation parameters are summarized in table 1 and table 2 .

Table1.Simulation parameters ofaggregating case.

\begin{tabular}{cc}
\hline Node Placement & Random \\
No of agents & 10 \\
Dimension ofVOI & $40 \mathrm{~m}$ \\
Communication Range & $10 \mathrm{~m}$ \\
Sensing Range & $5 \mathrm{~m}$ \\
A & 1 \\
$\mathrm{~B}$ & 0.1 \\
$\sigma_{\mathrm{x}}$ & 5 \\
$\sigma_{\mathrm{y}}$ & 5 \\
$\sigma_{\mathrm{z}}$ & 5 \\
$\alpha$ & 9 \\
$\eta$ & 0.2 \\
$\varepsilon$ & 1 \\
\hline
\end{tabular}

Considering a network with abovementioned parameters, the agent's initial, intermediate and final positions are shown in Figure5. Due to high positional error coefficient, the agents organize themselves in a way that there is minimum overlapping between sensing volumes around a centre.For better understanding an overall view of all 3D pictures are shown in the figures. As it is seen the initial state of the network is so compact and sensing volume have overlaps but in the final state the surveyed volume by the network is maximized around a centre without losing connectivity of agents.

Table2.Simulation parameters forspreading case.

\begin{tabular}{|cc|}
\hline Node Placement & Random \\
No of agents & 10 \\
Dimension ofVOI & $100 \mathrm{~m}$ \\
\hline Communication Range & $10 \mathrm{~m}$ \\
Sensing Range & $5 \mathrm{~m}$ \\
A & 1 \\
$\mathrm{~B}$ & 0.001 \\
$\sigma_{\mathrm{x}}$ & 5 \\
$\sigma_{\mathrm{y}}$ & 5 \\
$\sigma_{\mathrm{z}}$ & 5 \\
$\alpha$ & 9.5 \\
$\eta$ & 0.2 \\
$\varepsilon$ & 1 \\
\hline
\end{tabular}




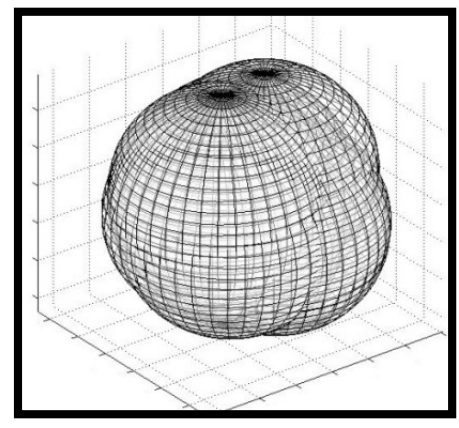

(a)

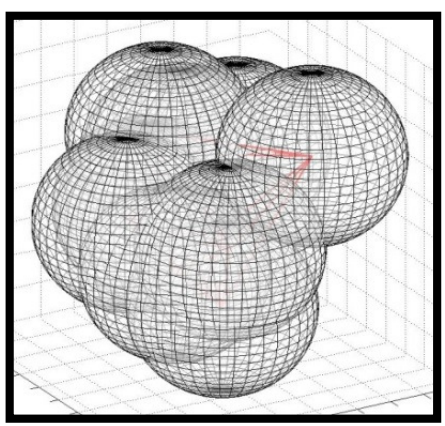

(c)

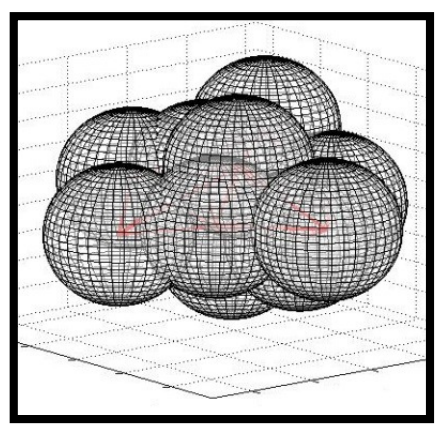

(e)

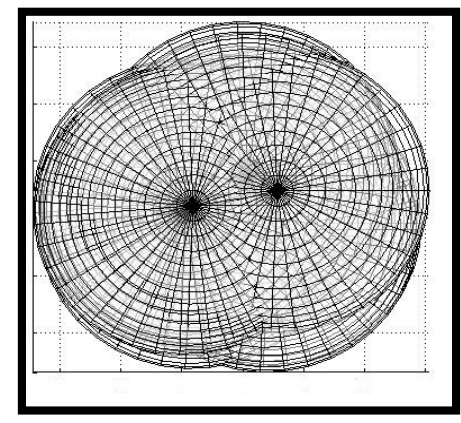

(b)

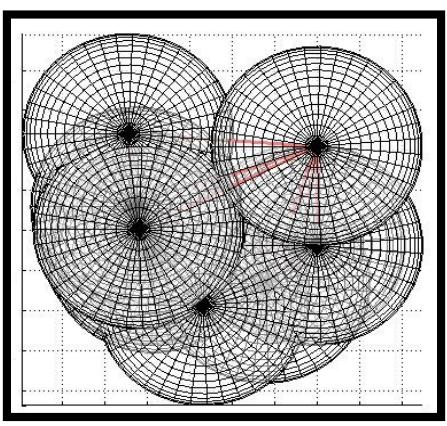

(d)

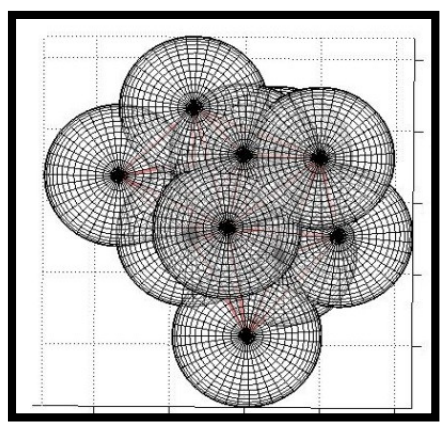

(f)

Figure 5. Deployment of agents in aggregating case (a, b) Lateral and top 3D view of initial deployment (c, d) Lateral and top 3D view of intermediate deployment (e, f) Lateral and top 3D view of final deployment.

Considering spreading case,total coverage is of network is some value near coverage upper bound i.e. $\mathcal{C}=\frac{\mathrm{n}}{6} \pi \mathrm{R}_{\mathrm{s}}^{3}$. In fact, by decreasing the positional error coefficient, the agents spread in the environment. In simulation process making the simulation more realistic agents are selected randomly to update their positions and the selected agent, updated its position with probability of $\% 95$ and it can be mentioned that all simulations converged to optimum solutions in below thirty iterations. In both cases sensing radii is considered half of communication radii which mean the agents with tangent sensing volumes are connected. Comparing this work to previous one [18], 
the algorithm try to herd the agents to centre while not getting disconnected and while maximizing coverage volume.

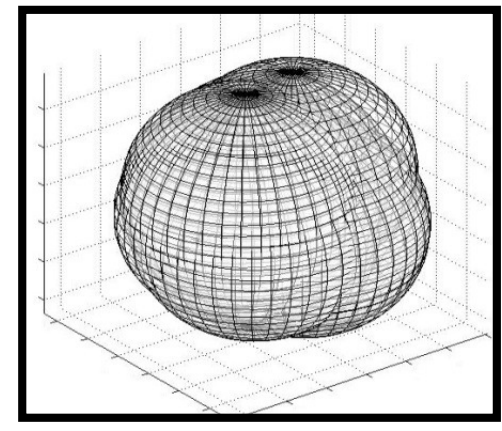

(a)

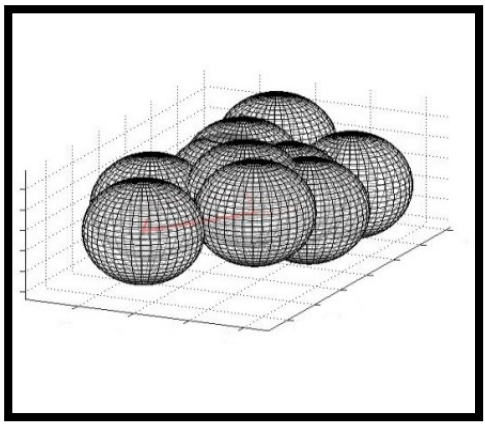

(c)

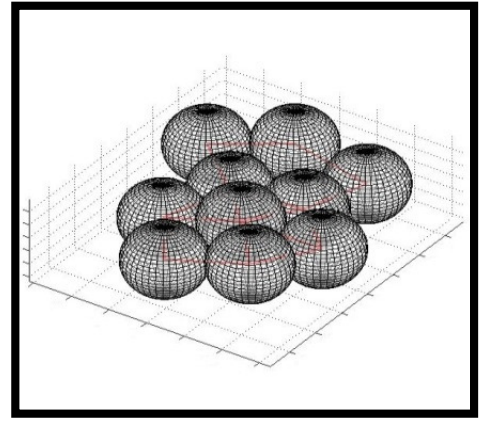

(e)

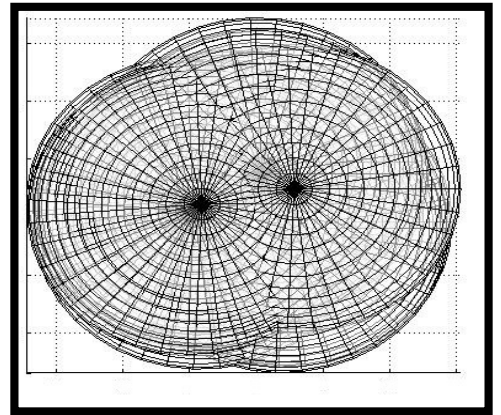

(b)

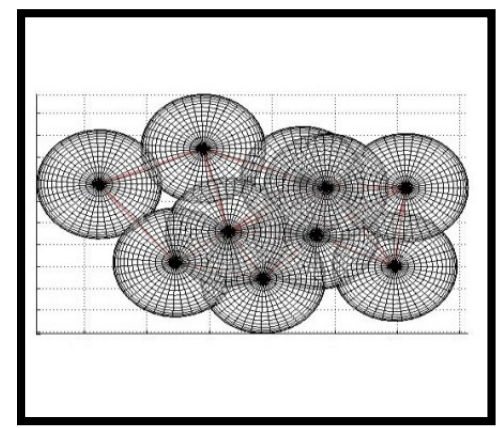

(d)

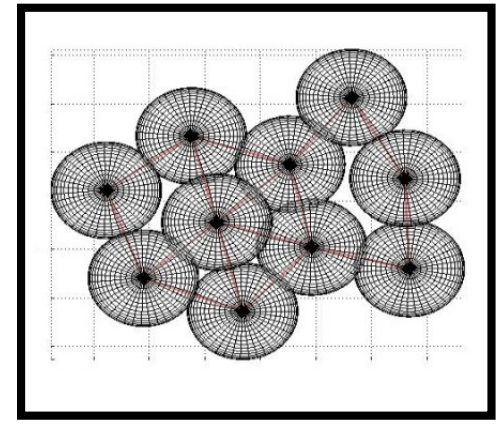

(f)

Figure 6. Deployment of agents in spreading case $(a, b)$ Lateral and top 3D view of initial deployment (c, d) Lateral and top 3D view of intermediate deployment(e, f) Lateral and top $3 \mathrm{D}$ view of final deployment.

\section{Conclusions}

In this paper a motion coordination algorithm for mobile agents deployed in a 3D wireless sensor network is proposed. It is assumed that agents have spherical shaped sensing and communication volume. The total coverage of network and quality of links can be controlled trough parameteroand as seen in simulations by adjustingonear sensing radii the approach would lead the network to a configuration with maximum connected coverage around predefined centre. In 
previous work [18],we proposed uniform importance for points in the environment but in this paperwe considered non-uniform requirement for sensing degree for regions of the environment and tried to drive agents to special points in the environment which are more important than the others Simulation results show the efficiency and fast convergence of algorithm.

\section{REFERENCES}

[1] “Aerial Common Sensor (acs)," http://www.globalsecurity.org/ intell/systems/acs.htm,2007.

[2] I. F. Akyildiz, D. Pompili, and T. Melodia, (2005) "Underwater Acoustic Sensor Networks: Research Challenges",Ad Hoc Networks (Elsevier), Vol. 3, No. 3, pp. 257-279.

[3] J. Kong, J.-h.Cui, D. Wu and M.Gerla(2005) "Building Underwater Ad-hoc Networks and Sensor Networks for Large Scale Real-time Aquatic Applications", IEEE Military Communications Conference (MILCOM'05), October 17-20, 2005. Atlantic City, New Jersey, USA.

[4] J.Heidemann, W. Ye, J. Wills, A. Syed, and Y. Li (2006) "Research Challenges and Applications for Underwater Sensor Networking”, IEEE Wireless Communications and Networking Conference, Las Vegas, Nevada, USA.

[5] I. Vasilescu, K. Kotay, D. Rus, M. Dunbabin and P. Corke, (2005) "Data Collection, Storage, and Retrieval with an Underwater Sensor Network", in Proc. $3^{\text {rd }}$ international conference on Embedded networked sensor systems (ACMSenSys'05), November 2-4, San Diego, California, USA, pp. 154165.

[6] J. Partan, J. Kurose, and Brian N. Levine (2007) "A Survey of Practical Issues in Underwater Networks",SIGMOBILE Mob.Comput.Commun. Rev., Vol. 11, No. 4, pp. 23-33.

[7] W. Tsujita, A. Yoshino, H. Ishida and T. Moriizumi, (2005 )"Gas Sensor Network for Air-Pollution Monitoring”,Sensors and Actuators B: Chemical, Vol. 110, No. 2, pp. 304-311.

[8] C.-F. Huang and Y.-C. Tseng (2003) "The Coverage Problem in a Wireless Sensor Network", inProc. of $2^{\text {nd }}$ ACM international conference on wireless sensor networks and applications (WSNA '03), New York, NY, USA, pp. 115-121.

[9] Y.-C. Wang, C.-C.Hu and Y.-C. Tseng (2005 )“Efficient Deployment Algorithms for Ensuring Coverage and Connectivity of Wireless Sensor Networks",in Proc. of the Wireless Internet Conference (WICON), pp. 114-121.

[10] X. Bai, D. Xuan, Z. Yun, and T. H. Lai (2008) "Complete Optimal Deployment Patterns for FullCoverage and k-Connectivity $(\mathrm{k} \leq 6)$ Wireless Sensor Networks", inProc. of the $9^{\text {th }}$ ACM MobiHoc, pp. 401-410.

[11] X. Bai, S. Kumar, D. Xuan, Z. Yun, and T. H. Lai (2006) "Deploying Wireless Sensors to Achieve Both Coverage and Connectivity", inProc. of the 7thACM international symposium on Mobile ad hoc networking and computing(ACM MobiHoc), New York, NY, USA, pp. 131-142.

[12] C.-F. Huang, Y.-C.Tseng, and L.-C. Lo (2004) "The Coverage Problem in Three- Dimensional Wireless Sensor Networks", in Proc. of IEEE GlobeCom 2004, pp. 3182-3186.

[13] S. Megerian and M. Potkonjak, (2003) "Low Power 0/1 Coverage and Scheduling Techniques in Sensor Networks",Technical Report 030001, University of California, Los Angeles, USA.

[14] FabiolaG. Nakamura, FredericoP. Quintao, Gustavo C. Menezes, and Geraldo R. Mateus(2005) "An Optimal Node Scheduling for Flat Wireless Sensor Networks", inProc.of the IEEE International Conference on Networking (ICN05), Vol. 3420, pp. 475-483.

[15] ILOG. ILOG CPLEX. Source: http://www.ilog.com/products/cplex/,May 2006.

[16] Y.stergiopoulos and A.Tzes(2011) "Coverage-oriented Coordination of Mobile Heterogeneous Networks", in Proc. of the $19^{\text {th }}$ Mediterranean Conference on control and Automation, June 20-23, pp. 175-180.

[17] Y.stergiopoulos and A.Tzes, (2011) "Decentralized Swarm Coordination: A Combined Coverage/Connectivity Approach", Journal of Intelligent \&Robotic Systems, Vol. 64, No. 3-4, pp. 603-623.

[18] Mohammad J. Heydari and S.Pashazadeh,(2012) "Connectivity Preserving Distributed Maximizing Coverage Algorithm for Three Dimensional Mobile Sensor Networks", International Journal of Advanced Research in Computer and Communication Engineering (IJARCCE), Vol. 1, Issue 5, July 2012, pp. 353-358.

[19] X. Bai, Z. Yun, D. Xuan, W. Jia, and W. Zhao, (2010) "Pattern Mutation in Wireless Sensor Deployment", in Proc. of IEE INFOCOM, pp. 1-9. 
[20] S. M. N. Alam and Zygmunt J. Haas (2006) "Coverage and Connectivity in Three-Dimensional Networks", inProc. of $12^{\text {th }}$ annual international conference on Mobile computing and networking (MobiCom 2006), pp.346-357.

[21] X. Bai, C. Zhang, D. Xuan, and W. Jia (2009) "Full-Coverage and k-Connectivity $(\mathrm{k}=14,6)$ Three Dimensional Networks", inProc. of INFOCOM 2009, pp. 388-396.

[22] X. Bai, C. Zhang, D. Xuan, J. Teng, and W. Jia (2009) "Low-Connectivity and Full-Coverage Three Dimensional Wireless Sensor Networks", inProc. of $10^{\text {th }}$ ACM international symposium on Mobile ad hoc networking and computing(MobiHoc 2009), pp. 145-154.

[23] C.-H. Wu, K.-C.Lee, and Y.-C. Chung (2007) "A Delaunay Triangulation Based Method for Wireless Sensor Network Deployment”,Computer Communications, Vol. 30, No. 14-15, pp.2744-2752.

[24] M.A.M. Vieira, L.F.M. Vieira, L.B. Ruiz, A.A.F. Loureiro, A.O. Fernandes, and J.M.S. Nogueira, (2003) "Scheduling Nodes in Wireless Sensor Networks: A Voronoi Approach", in Proc. of the $28^{\text {th }}$ Annual IEEE International Conference on Local Computer Networks (LCN' 03), pp. 423-429.

[25] K. Akkaya, S.Janapala(2008) "Maximizing Connected Coverage via Controlled Actor Relocation in Wireless Sensor and Actor Networks", Computer Networks (Elsevier),Vol. 52, No. 14, pp. 27792796.

[26] G. Wang,G. Cao, Tom L. Porta(2006)“Movement-Assisted Sensor Deployment”, IEEE Transactions on Mobile Computing (ToMC), Vol. 5, No. 6, pp. 640-652.

[27] Y. Zou, K. Chakrabarty(2004) "Sensor Deployment and Target Localization in Distributed Sensor Networks",ACM Transactions on Embedded Computing Systems, Vol. 3, No. 1, pp. 61-91.

[28] O.Dengiz, A.Konak and Alice E. Smith, (2011) "Connectivity Management in Mobile Ad-hoc Networks Using Particle Swarm Optimization”,Ad Hoc Networks, Vol.9, No. 7, pp. 1312-1326.

[29] J.-W.Lee, B.-S.Choi, and J.-J. Lee, (2011) "Energy-Efficient Coverage of Wireless Sensor Networks Using Ant Colony Optimization with Three Types of Pheromones",IEEE Transactions on Industrial Informatics, Vol. 7, No. 3, pp. 419-427.

\section{Authors}

Mohammad Javad Heydari received his B.Sc. in Computer Engineering from shahed University of Tehran in 2009.He is currently pursuingthe M.Sc. degree in Artificial Intelligence at University of Tabriz in Iran. His research interests mainly include Wireless Sensor Networks, Multi-Robot Systems and Swarm Intelligence.

Saeid Pashazadeh is Assistant Professor of Software Engineering and chair of Information Technology Department at Faculty of Electrical and Computer Engineering in University of Tabriz in Iran. He received his B.Sc. in Computer Engineering from Sharif Technical University of Iran in 1995. He obtained M.Sc. and Ph.D. in Computer Engineering from Iran University of Science and Technology in Iran in 1998, 2010 respectively.He was Lecturer in Faculty of Electrical Engineering in Sahand University of Technology in Iran from 1999 until 2004. His main interest is in

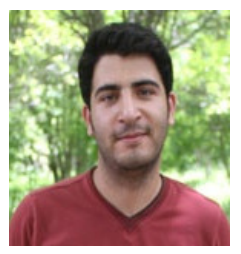
the development, modeling and formal verification of distributed systems, and computer security and wireless sensor and wireless actor networks. He is member of IEEE and senior member of IACSIT. 